\title{
An Accelerated Homotopy Perturbation Method for Solving Nonlinear Two-Dimensional Volterra-Fredholm Integrodifferential Equations
}

\author{
F. A. Hendi ${ }^{1}$ and M. M. Al-Qarni ${ }^{2}$ \\ ${ }^{1}$ Department of Mathematics, Faculty of Science, King Abdulaziz University, Jeddah, Saudi Arabia \\ ${ }^{2}$ Department of Mathematics, Faculty of Science, King Khalid University, Abha, Saudi Arabia
}

Correspondence should be addressed to F. A. Hendi; falhendi@kau.edu.sa

Received 7 January 2017; Revised 19 April 2017; Accepted 30 April 2017; Published 30 July 2017

Academic Editor: Xiao-Jun Yang

Copyright (c) 2017 F. A. Hendi and M. M. Al-Qarni. This is an open access article distributed under the Creative Commons Attribution License, which permits unrestricted use, distribution, and reproduction in any medium, provided the original work is properly cited.

\begin{abstract}
We propose and apply coupling of the variational iteration method (VIM) and homotopy perturbation method (HPM) to solve nonlinear mixed Volterra-Fredholm integrodifferential equations (VFIDE). In this approach, we use a new formula called variational homotopy perturbation method (VHPM) and variational accelerated homotopy perturbation method (VAHPM). This approach is based on the form of He's polynomials and on a new form of He's polynomials. We discuss the convergence of the technique. Some numerical examples are introduced to verify the efficiency of this technique.
\end{abstract}

\section{Introduction}

In recent years, there has been a clear interest in integrodifferential equations which are a combination of differential and Volterra-Fredholm integral equations. Integrodifferential equations play an important role in many branches of linear and nonlinear functional analyses and their applications. The mentioned integrodifferential equations are usually difficult to solve analytically, so approximation strategies are required to obtain the solution of the linear and nonlinear integrodifferential equations [1].

Many researchers studied and discussed the linear VFIDE [2]. Al-Jubory [3] introduced some approximation methods to solve Volterra-Fredholm integral and integrodifferential equations. Dadkhah et al. in [4] used a numerical solution of nonlinear VFIDE by using Legendre wavelets. Rabbani and Kiasoltani [5] studied the solving of a nonlinear system of VFIDE by using the discrete collocation method. Gherjalar and Mohammadikia [6] solved integral and integrodifferential equations by using the B-splines function. In this work, we used the HPM and VIM to solve the two-dimensional nonlinear VFIDE as follows:

$$
\begin{aligned}
& \sum_{j=0}^{k} P_{j}\left(x_{1}, \jmath_{1}\right) u^{j}\left(x_{1}, \jmath_{1}\right) \\
& =\dot{f}\left(x_{1}, \jmath_{1}\right) \\
& \quad+\int_{a}^{x_{1}} \int_{\Omega} F\left(x_{1}, \jmath_{1}, y, \tau\right) \gamma\left(u^{l}(y, \tau)\right) d y d \tau, \\
& \quad\left(x_{1}, \jmath_{1}\right) \in \dot{J}=\left[a, x_{1}\right] \times \Omega,
\end{aligned}
$$

with initial conditions

$$
u^{r}\left(a, \jmath_{1}\right)=g_{r}, \quad r=0,1, \ldots, k-1, \Omega=[a, b],
$$

where $u^{j}\left(x_{1}, \jmath_{1}\right)=d^{j} u / d x_{1}^{j}$. The functions $\dot{f}\left(x_{1}, \jmath_{1}\right)$, $F\left(x_{1}, j_{1}, y, \tau\right)$ and $\gamma\left(u^{l}(y, \tau)\right), l>0$, are analytic functions on $J^{\prime}$, and functions $P_{j}\left(x_{1}, j_{1}\right), j=0,1, \ldots, k, P_{k}\left(x_{1}, j_{1}\right) \neq 0$ are given. 
For VIM and HPM, which were proposed by $\mathrm{He}$ in $[7,8]$, the solution is considered as the summation of an infinite series which is assumed to be convergent to the exact solution. In recent years, HPM has been applied with great success, so relations and algorithms have been deduced and continuously improved to obtain an accurate solution for a large variety of linear and nonlinear problems. For instance, $\mathrm{He}$ in [7] used a strategy to solve some integrodifferential equations where he chose an initial approximate solution in the form of an exact solution with unknown constants.

In this paper, a new approach based on VIM with HPM is introduced to solve the two-dimensional nonlinear VFIDE.

\section{The HPM}

In this section, we will present the HPM. We consider a general integral equation

$$
L u=0 \text {, }
$$

where $L$ is an integral operator. Define a convex homotopy $\breve{H}(\vartheta, \wp)$ by

$$
\breve{H}(\vartheta, \wp)=(1-\wp) F(\vartheta)+\wp L(\vartheta)=0, \quad \wp \in[0,1],
$$

where $F(\vartheta)$ is a functional operator with solution $\vartheta_{0}$. Then,

$$
\begin{aligned}
& \breve{H}(\vartheta, 0)=\digamma(\vartheta)=0, \\
& \breve{H}(\vartheta, 1)=L(\vartheta)=0,
\end{aligned}
$$

and the process of changing $\varnothing$ from 0 to 1 is just that of changing $\vartheta$ from $\vartheta_{0}$ to $u$. In topology, this is called deformation, and $F(\vartheta)$ and $L(\vartheta)$ are called homotopies.

According to the HPM, we can use the embedding parameter $\wp$ as a "small parameter" and assume that the solution of (4) can be written as a power series in $\wp$ :

$$
\vartheta=u_{0}+\wp u_{1}+\wp^{2} u_{2}+\cdots=\sum_{i=0}^{\infty} \wp^{i} u_{i}=u .
$$

When $\wp \rightarrow 1$, the approximate solution of (3) is obtained with

$$
u=\lim _{\wp \rightarrow 1} \vartheta=u_{0}+u_{1}+u_{2}+\cdots=\sum_{i=0}^{\infty} u_{i} .
$$

Series (7) is convergent for most cases; however, the rate of convergence depends upon the nonlinear operator $L$ [9].

\section{The HPM for Solving Nonlinear Mixed VFIDE}

In what follows, we display an outline for utilizing the HPM for solving the nonlinear VFIDE. Equation (1) can be written as follows:

$$
\begin{aligned}
& u\left(x_{1}, \jmath_{1}\right) \\
& =L^{-1}\left(\frac{\dot{f}\left(x_{1}, \jmath_{1}\right)}{P_{k}\left(x_{1}, \jmath_{1}\right)}\right)+\sum_{r=0}^{k-1} \frac{1}{(r !)}\left(x_{1}-\jmath_{1}\right)^{r} g_{r}
\end{aligned}
$$

$$
\begin{aligned}
& +L^{-1}\left(\int_{a}^{x_{1}} \int_{\Omega} \frac{F\left(x_{1}, \jmath_{1}, y, \tau\right) \gamma\left(u^{l}(y, \tau)\right)}{P_{k}\left(x_{1}, \jmath_{1}\right)} d y d \tau\right) \\
& -L^{-1}\left(\sum_{j=0}^{k-1} \frac{P_{j}\left(x_{1}, \jmath_{1}\right)}{P_{k}\left(x_{1}, \jmath_{1}\right)} u^{j}\left(x_{1}, \jmath_{1}\right)\right),
\end{aligned}
$$

where $L^{-1}$ is the multiple integration operator given as follows:

$$
L^{-1}(\cdot)=\int_{a}^{x_{1}} \int_{a}^{x_{1}} \cdots \int_{a}^{x_{1}}(\cdot) d x_{1} d x_{1} \cdots d x_{1}
$$

( $n$ times).

So, (8) takes the form

$$
\begin{aligned}
& u\left(x_{1}, \jmath_{1}\right)=L^{-1}\left(\frac{\dot{f}\left(x_{1}, \jmath_{1}\right)}{P_{k}\left(x_{1}, \jmath_{1}\right)}\right)+\sum_{r=0}^{k-1} \frac{1}{(r !)}\left(x_{1}-\jmath_{1}\right)^{r} g_{r} \\
& +\int_{a}^{x_{1}} \int_{\Omega} \frac{\left(x_{1}-\jmath_{1}\right)^{k}}{(k) !} \frac{F\left(x_{1}, \jmath_{1}, y, \tau\right) \gamma\left(u^{l}(y, \tau)\right)}{P_{k}\left(x_{1}, \jmath_{1}\right)} d y d \tau \\
& -\sum_{j=0}^{k-1} \int_{a}^{x_{1}} \frac{\left(x_{1}-\jmath_{1}\right)^{k-1}}{(k-1) !} \frac{P_{j}\left(x_{1}, \jmath_{1}\right)}{P_{k}\left(x_{1}, \jmath_{1}\right)} u^{j}\left(x_{1}, \jmath_{1}\right) d \jmath_{1}
\end{aligned}
$$

since

$$
\begin{aligned}
& \sum_{j=0}^{k-1} L^{-1}\left(\frac{P_{j}\left(x_{1}, \jmath_{1}\right)}{P_{k}\left(x_{1}, \jmath_{1}\right)}\right) u^{j}\left(x_{1}, \jmath_{1}\right) \\
& \quad=\sum_{j=0}^{k-1} \int_{a}^{x_{1}} \frac{\left(x_{1}-\jmath_{1}\right)^{k-1}}{(k-1) !} \frac{P_{j}\left(x_{1}, \jmath_{1}\right)}{P_{k}\left(x_{1}, \jmath_{1}\right)} u^{j}\left(x_{1}, \jmath_{1}\right) d \jmath_{1} .
\end{aligned}
$$

To illustrate the HPM, for nonlinear mixed VFIDE, let us consider (8):

$$
\begin{aligned}
& \breve{H}(\vartheta, \wp)=\vartheta\left(x_{1}, \jmath_{1}\right)-L^{-1}\left(\frac{\dot{f}\left(x_{1}, \jmath_{1}\right)}{P_{k}\left(x_{1}, \jmath_{1}\right)}\right)-\sum_{r=0}^{k-1} \frac{1}{(r !)}\left(x_{1}\right. \\
& \left.-\jmath_{1}\right)^{r} g_{r} \\
& -\wp\left[L^{-1}\left(\int_{a}^{x_{1}} \int_{\Omega} \frac{F\left(x_{1}, \jmath_{1}, y, \tau\right) \gamma\left(\vartheta^{l}(y, \tau)\right)}{P_{k}\left(x_{1}, \jmath_{1}\right)} d y d \tau\right)\right. \\
& \left.-L^{-1}\left(\sum_{j=0}^{k-1} \frac{P_{j}\left(x_{1}, \jmath_{1}\right)}{P_{k}\left(x_{1}, \jmath_{1}\right)} \vartheta^{j}\left(x_{1}, \jmath_{1}\right)\right)\right]=0 .
\end{aligned}
$$

By the HPM, we can expand $\vartheta\left(x_{1}, \jmath_{1}\right)$ into the form

$$
\begin{gathered}
\vartheta\left(x_{1}, \jmath_{1}\right)=u_{0}\left(x_{1}, \jmath_{1}\right)+\wp u_{1}\left(x_{1}, \jmath_{1}\right)+\wp^{2} u_{2}\left(x_{1}, \jmath_{1}\right) \\
+\cdots=\sum_{i=0}^{\infty} \wp^{i} u_{i}\left(x_{1}, \jmath_{1}\right)=u\left(x_{1}, \jmath_{1}\right) .
\end{gathered}
$$

When $\wp \rightarrow 1$, the approximate solution is obtained with

$$
\begin{aligned}
u\left(x_{1}, \jmath_{1}\right) & =\lim _{\wp \rightarrow 1} \vartheta\left(x_{1}, \jmath_{1}\right) \\
& =u_{0}\left(x_{1}, \jmath_{1}\right)+u_{1}\left(x_{1}, \jmath_{1}\right)+u_{2}\left(x_{1}, \jmath_{1}\right)+\cdots
\end{aligned}
$$


and in sum, according to [10], He's HPM considers the nonlinear term $\gamma(u)$ as

$$
\gamma(u)=\sum_{i=0}^{\infty} \wp^{i} \breve{H}_{i}=\breve{H}_{0}+\wp \breve{H}_{1}+\wp^{2} \breve{H}_{2}+\cdots,
$$

where $H_{n}$ 's are the so-called He's polynomials [10], which can be calculated by using the formula

$$
\breve{H}_{n}=\frac{1}{n} \frac{\partial^{n}}{\partial \wp^{n}}\left[\gamma\left(\sum_{i=0}^{\infty} \wp^{i} u_{i}\right)\right]_{\wp=0}, \quad n=0,1,2, \ldots
$$

Substituting (13) and (15) into (12) and equating the terms with identical powers of $\wp$, we have

$$
\begin{aligned}
& \wp^{0}: u_{0}\left(x_{1}, \jmath_{1}\right) \\
&= L^{-1}\left(\frac{\dot{f}\left(x_{1}, \jmath_{1}\right)}{P_{k}\left(x_{1}, \jmath_{1}\right)}\right)+\sum_{r=0}^{k-1} \frac{1}{(r !)}\left(x_{1}-\jmath_{1}\right)^{r} g_{r}, \\
& \wp^{i+1}: u_{i+1}\left(x_{1}, \jmath_{1}\right) \\
&=L^{-1}\left(\int_{a}^{x_{1}} \int_{\Omega} \frac{F\left(x_{1}, \jmath_{1}, y, \tau\right)}{P_{k}\left(x_{1}, \jmath_{1}\right)} \breve{H}_{i} d y d \tau\right) \\
& \quad-L^{-1}\left(\sum_{j=0}^{k-1} \frac{P_{j}\left(x_{1}, \jmath_{1}\right)}{P_{k}\left(x_{1}, \jmath_{1}\right)} L_{i_{j}}\right), \quad i \geq 0 .
\end{aligned}
$$

The nonlinear terms $\gamma\left(u^{l}\left(x_{1}, j_{1}\right)\right)$ and $D^{j}\left(u\left(x_{1}, j_{1}\right)\right)\left(D^{j}=\right.$ $\partial^{j} u\left(x_{1}, j_{1}\right) / \partial x_{1}^{j}$ is a derivative operator) are usually represented by an infinite series of the so-called He's polynomials as follows:

$$
\begin{aligned}
\gamma\left(u^{l}\left(x_{1}, \jmath_{1}\right)\right) & =\sum_{i=0}^{\infty} \breve{H}, \\
D^{j}\left(u\left(x_{1}, \jmath_{1}\right)\right) & =\sum_{i=0}^{\infty} L_{i_{j}} .
\end{aligned}
$$

The components $u_{i}\left(x_{1}, j_{1}\right), i \geq 0$, can be computed by using the recursive relations (17).

\section{A New Formula to He's Polynomials}

He's polynomials are not unique; another formula of He's polynomials $\left(\breve{H}_{n}\right)$, called accelerated He's polynomials, is represented by $\left(\bar{H}_{n}\right)$; in [11], the author proved that

$$
\gamma(u)=\sum_{n=0}^{\infty} \breve{H}_{n}=\sum_{n=0}^{\infty} \bar{H}_{n}
$$

in which $\breve{H}_{n}$ can be written in the new mathematical form

$$
\bar{H}_{n}=\gamma\left(\delta_{n}\right)-\sum_{i=0}^{n-1} \bar{H}_{i}
$$

where the partial sum $\delta_{n}=\sum_{i=0}^{n} u_{i}\left(x_{1}, \jmath_{1}\right)$ and $\bar{H}_{0}=$ $\gamma\left(u_{0}\right)$. Substituting (13) and $\gamma(u)=\sum_{n=0}^{\infty} \wp^{n} \bar{H}_{n}$ into (12) and equating the terms with identical powers of $\wp$, we obtain the following accelerated recursive formula:

$$
\begin{aligned}
\wp^{0}: & u_{0}\left(x_{1}, \jmath_{1}\right) \\
= & L^{-1}\left(\frac{\dot{f}\left(x_{1}, \jmath_{1}\right)}{P_{k}\left(x_{1}, \jmath_{1}\right)}\right)+\sum_{r=0}^{k-1} \frac{1}{(r !)}\left(x_{1}-\jmath_{1}\right)^{r} g_{r}, \\
\wp^{i+1}: & u_{i+1}\left(x_{1}, \jmath_{1}\right) \\
= & L^{-1}\left(\int_{a}^{x_{1}} \int_{\Omega} \frac{F\left(x_{1}, \jmath_{1}, y, \tau\right)}{P_{k}\left(x_{1}, \jmath_{1}\right)} \bar{H}_{i} d y d \tau\right) \\
& \quad-L^{-1}\left(\sum_{j=0}^{k-1} \frac{P_{j}\left(x_{1}, j_{1}\right)}{P_{k}\left(x_{1}, \jmath_{1}\right)} L_{i_{j}}\right), \quad i \geq 0 .
\end{aligned}
$$

For example, if $\gamma(u)=u^{3}$, the first four polynomials using formulas (16) and (20) are computed to be as follows.

Using formula (16),

$$
\begin{aligned}
& \breve{H}_{0}=u_{0}^{3} . \\
& \breve{H}_{1}=3 u_{0}^{2} u_{1} . \\
& \breve{H}_{2}=3 u_{0} u_{1}^{2}+3 u_{0}^{2} u_{2} . \\
& \breve{H}_{3}=u_{1}^{3}+6 u_{0} u_{1} u_{2}+3 u_{0}^{2} u_{3} . \\
& \breve{H}_{4}=3 u_{1}^{2} u_{2}+3 u_{0} u_{2}^{2}+6 u_{0} u_{1} u_{3}+3 u_{0}^{2} u_{4} .
\end{aligned}
$$

Using formula (20),

$$
\begin{aligned}
\bar{H}_{0}= & u_{0}^{3} \cdot \\
\bar{H}_{1}= & 3 u_{0}^{2} u_{1}+3 u_{0} u_{1}^{2}+u_{1}^{3} \cdot \\
\bar{H}_{2}= & 3 u_{0}^{2} u_{2}+3 u_{0} u_{2}^{2}+3 u_{1}^{2} u_{2}+3 u_{1} u_{2}^{2}+6 u_{0} u_{1} u_{2} \\
& +u_{2}^{3} . \\
\bar{H}_{3}= & 3 u_{0}^{2} u_{3}+3 u_{0} u_{3}^{2}+3 u_{1}^{2} u_{3}+3 u_{1} u_{3}^{2}+3 u_{2}^{2} u_{3} \\
& +3 u_{2} u_{3}^{2}+6 u_{0} u_{1} u_{3}+6 u_{0} u_{2} u_{3}+6 u_{1} u_{2} u_{3} \\
& +u_{3}^{3} . \\
\bar{H}_{4}= & 3 u_{0}^{2} u_{4}+3 u_{0} u_{4}^{2}+3 u_{1}^{2} u_{4}+3 u_{1} u_{4}^{2}+3 u_{2}^{2} u_{4} \\
& +3 u_{2} u_{4}^{2}+3 u_{3}^{2} u_{4}+3 u_{3} u_{4}^{2}+6 u_{0} u_{1} u_{4} \\
& +6 u_{0} u_{2} u_{4}+6 u_{0} u_{3} u_{4}+6 u_{1} u_{2} u_{4}+6 u_{1} u_{3} u_{4} \\
& +6 u_{2} u_{3} u_{4}+u_{4}^{3} .
\end{aligned}
$$

Clearly, the first four polynomials computed using the suggested formula (20) include the first four polynomials computed using formula (16) in addition to other terms that should appear in $\breve{H}_{5}, \breve{H}_{6}, \breve{H}_{7}, \ldots$ using formula (16). Thus, 
the solution that was obtained using formula (20) enforces many terms to the calculation processes earlier, yielding faster convergence.

\section{The VIM}

Consider the differential equation

$$
u+\aleph u=\dot{f}\left(x_{1}\right),
$$

where $\imath$ is a linear operator, $\aleph$ is a nonlinear operator, and $\dot{f}\left(x_{1}\right)$ is a given continuous function. The VIM presents a correction functional for (24) in the form

$$
\begin{aligned}
u_{i+1}\left(x_{1}\right)= & u_{i}\left(x_{1}\right) \\
& +\int_{0}^{x_{1}} \lambda(\varsigma)\left(\imath u_{i}(\varsigma)+\aleph \tilde{u}_{i}(\varsigma)-\dot{f}(\varsigma)\right) d \varsigma,
\end{aligned}
$$

where $\lambda$ is a Lagrange multiplier $[8,9]$ which can be identified optimally via variational theory, $u_{i}$ is the $n$th approximate solution, and $\widetilde{u}_{i}$ denotes a restricted variation (i.e., $\varrho u_{i}=0$ ).

\section{Adapting VIM with HPM for Solving (1) and (2)}

This modified version of VHPM is obtained by the coupling of VIM with HPM. First, by using formula (16), we obtain

$$
\begin{gathered}
\sum_{i=0}^{\infty} \wp^{i} u_{i+1}\left(x_{1}, \jmath_{1}\right)=u_{i}\left(x_{1}, \jmath_{1}\right)+\wp \int_{0}^{j_{1}} \lambda(\varsigma) \\
\cdot\left[\sum_{i=0}^{\infty} \wp^{i} u_{i}^{k}\left(x_{1}, \varsigma\right)-\frac{\dot{f}\left(x_{1}, \varsigma\right)}{P_{k}\left(x_{1}, \varsigma\right)}\right. \\
-\int_{a}^{x_{1}} \int_{\Omega} \frac{F\left(x_{1}, \varsigma, y, \tau\right)}{P_{k}\left(x_{1}, \varsigma\right)} \sum_{i=0}^{\infty} \wp^{i} \breve{H}_{i} d y d \tau \\
\left.+\sum_{j=0}^{k-1} \frac{P_{j}\left(x_{1}, \varsigma\right)}{P_{k}\left(x_{1}, \varsigma\right)}\left(\sum_{i=0}^{\infty} \wp^{i} u_{i}^{j}\left(x_{1}, \varsigma\right)\right)\right] d \varsigma
\end{gathered}
$$

which is called VHPM.

Second, by using formula (20),

$$
\begin{gathered}
\sum_{i=0}^{\infty} \wp^{i} u_{i+1}\left(x_{1}, \jmath_{1}\right)=u_{i}\left(x_{1}, \jmath_{1}\right)+\wp \int_{0}^{\jmath_{1}} \lambda(\varsigma) \\
\cdot\left[\sum_{i=0}^{\infty} \wp^{i} u_{i}^{k}\left(x_{1}, \varsigma\right)-\frac{\dot{f}\left(x_{1}, \varsigma\right)}{P_{k}\left(x_{1}, \varsigma\right)}\right. \\
-\int_{a}^{x_{1}} \int_{\Omega} \frac{F\left(x_{1}, \varsigma, y, \tau\right)}{P_{k}\left(x_{1}, \varsigma\right)} \sum_{i=0}^{\infty} \wp^{i} \bar{H}_{i} d y d \tau \\
\left.+\sum_{j=0}^{k-1} \frac{P_{j}\left(x_{1}, \varsigma\right)}{P_{k}\left(x_{1}, \varsigma\right)}\left(\sum_{i=0}^{\infty} \wp^{i} u_{i}^{j}\left(x_{1}, \varsigma\right)\right)\right] d \varsigma
\end{gathered}
$$

which is called VAHPM.
The following is the algorithm for calculating $u_{0}, u_{1}$, $u_{2}, \ldots, u_{n-1}, u_{n}$ :

Step 1: input nonlinear term $\gamma\left(u^{l}\right)$ and $n$ that is the order of He's polynomials, endpoint $a, b$, initial conditions $g_{1}, g_{2}, \ldots, g_{r}$, free term, and $F\left(x_{1}, \jmath_{1}, y, \tau\right)$.

Step 2: set $u=u_{0}+\wp u_{1}+\wp^{2} u_{2}+\cdots+\wp^{n} u_{n}$.

Step 3: let $\sum_{k=0}^{n} \wp^{k} \breve{H}_{k}=\gamma\left(u_{0}+\wp u_{1}+\wp^{2} u_{2}+\cdots+\wp^{n} u_{n}\right)$.

Step 4: For $i=0,1, \ldots, n$, do

(a) ith-order derivative of both sides of the equality with respect to $\wp$ :

$$
\begin{aligned}
& \frac{\partial^{i}\left(\sum_{k=0}^{n} \wp^{k} \breve{H}_{k}\right)}{\partial \wp^{i}} \\
& \quad=\frac{\partial \gamma\left(u_{0}+\wp u_{1}+\wp^{2} u_{2}+\cdots+\wp^{n} u_{n}\right)}{\partial \wp^{i}} ;
\end{aligned}
$$

(b) let $\wp=0$ of the above equality and determine $\breve{H}_{i}$ by solving the equation with respect to $\breve{H}_{i}$.

End do.

Step 5: put $u_{0}=$ initial conditions.

Step 6: for $i=1, \ldots, n$, do

Step 7: calculate $u_{i}$ by applying (26),

end do.

Step 8: set $u=\lim _{i \rightarrow \infty} u_{i}$ as the approximate of the exact solution.

\section{Convergence Analysis}

In this section, the sufficient condition that guarantees the existence of a unique solution is introduced in Theorem 1, convergence of the methods is proved in Theorems 2 and 3 , and finally the maximum absolute error of the truncated series $\left(u_{i}\left(x_{1}, \jmath_{1}\right)=\sum_{i=0}^{\infty} u_{i}\left(x_{1}, \jmath_{1}\right)\right)$ is estimated in Theorem 4 .

Considering (10), we set

$$
\begin{aligned}
& L^{-1}\left(\frac{\dot{f}\left(x_{1}, \jmath_{1}\right)}{P_{k}\left(x_{1}, \jmath_{1}\right)}\right)+\sum_{r=0}^{k-1} \frac{1}{(r !)}\left(x_{1}-\jmath_{1}\right)^{r} g_{r} \\
& \quad=f_{1}\left(x_{1}, \jmath_{1}\right), \\
& k_{1}\left(x_{1}, \jmath_{1}\right)=\int_{\Omega} \frac{\left(x_{1}-\jmath_{1}\right)^{k}}{(k) !} \frac{F\left(x_{1}, \jmath_{1}, y, \tau\right)}{P_{k}\left(x_{1}, \jmath_{1}\right)} d y \\
& k_{2}\left(x_{1}, \jmath_{1}\right)=\frac{\left(x_{1}-\jmath_{1}\right)^{k-1}}{(k-1) !} \frac{P_{j}\left(x_{1}, \jmath_{1}\right)}{P_{k}\left(x_{1}, \jmath_{1}\right)} .
\end{aligned}
$$


We can write (10) as

$$
\begin{aligned}
u\left(x_{1}, \jmath_{1}\right)= & f_{1}\left(x_{1}, \jmath_{1}\right) \\
& +\int_{a}^{x_{1}} k_{1}\left(x_{1}, \jmath_{1}\right) \gamma\left(u^{l}(y, \tau)\right) d \tau- \\
& \cdot \sum_{j=0}^{k-1} \int_{a}^{x_{1}} k_{2}\left(x_{1}, \jmath_{1}\right) u^{j}\left(x_{1}, \jmath_{1}\right) d \jmath_{1} .
\end{aligned}
$$

We assume $f_{1}\left(x_{1}, \jmath_{1}\right)$ is bounded for all $x_{1}, \jmath_{1}$ in $J$ and

$$
\begin{aligned}
& \left|k_{1}\left(x_{1}, \jmath_{1}\right)\right| \leq N_{1} \\
& \left|k_{2}\left(x_{1}, \jmath_{1}\right)\right| \leq N_{1_{j}}, \quad j=0,1, \ldots, k-1, \forall x_{1}, \jmath_{1} \in \dot{J} .
\end{aligned}
$$

Also, we suppose the nonlinear terms $\gamma\left(u^{l}\left(x_{1}, j_{1}\right)\right)$ and $D^{j}\left(u\left(x_{1}, \jmath_{1}\right)\right)$ are Lipschitz continuous with

$$
\begin{gathered}
\left|\gamma\left(u^{(l)}\left(x_{1}, \jmath_{1}\right)\right)-\gamma\left(u^{(l)^{*}}\left(x_{1}, \jmath_{1}\right)\right)\right| \\
\leq d\left|u\left(x_{1}, \jmath_{1}\right)-u^{*}\left(x_{1}, \jmath_{1}\right)\right| \\
\left|D^{j}\left(u\left(x_{1}, \jmath_{1}\right)\right)-D^{j}\left(u^{*}\left(x_{1}, \jmath_{1}\right)\right)\right| \\
\leq Z_{j}\left|u\left(x_{1}, \jmath_{1}\right)-u^{*}\left(x_{1}, \jmath_{1}\right)\right|,
\end{gathered}
$$

$$
j=0,1, \ldots, k-1 \text {. }
$$

Hence, we set

$$
\begin{aligned}
& \Gamma=(b-a)\left(d N_{1}+k Z N\right), \\
& Z=\max \left|Z_{j}\right|, \\
& N=\max \left|N_{1_{j}}\right|,
\end{aligned}
$$

$$
j=0,1, \ldots, k-1 \text {. }
$$

Theorem 1. Two-dimensional nonlinear VFIDE has a unique solution whenever $0<\Gamma<1$.

Proof. Let $u$ and $u^{*}$ be two different solutions of (30). Then,

$$
\begin{aligned}
& \left|u\left(x_{1}, \jmath_{1}\right)-u^{*}\left(x_{1}, \jmath_{1}\right)\right|=\mid \int_{a}^{x_{1}} k_{1}\left(x_{1}, \jmath_{1}\right) \\
& \cdot\left[\gamma\left(u^{(l)}\left(x_{1}, \jmath_{1}\right)\right)-\gamma\left(u^{(l)^{*}}\left(x_{1}, \jmath_{1}\right)\right)\right] d \tau \\
& -\sum_{j=0}^{k-1} \int_{a}^{x_{1}} k_{2}\left(x_{1}, \jmath_{1}\right) \\
& \cdot\left[D^{j}\left(u\left(x_{1}, \jmath_{1}\right)\right)-D^{j}\left(u^{*}\left(x_{1}, \jmath_{1}\right)\right)\right] d \jmath_{1} \mid
\end{aligned}
$$

$$
\begin{aligned}
& \leq \int_{a}^{x_{1}}\left|k_{1}\left(x_{1}, \jmath_{1}\right)\right| \mid \gamma\left(u^{(l)}\left(x_{1}, \jmath_{1}\right)\right) \\
& -\gamma\left(u^{(l)^{*}}\left(x_{1}, \jmath_{1}\right)\right)\left|d \tau+\sum_{j=0}^{k-1} \int_{a}^{x_{1}}\right| k_{2}\left(x_{1}, \jmath_{1}\right) \mid \\
& \cdot\left|D^{j}\left(u\left(x_{1}, \jmath_{1}\right)\right)-D^{j}\left(u^{*}\left(x_{1}, \jmath_{1}\right)\right)\right| d \jmath_{1} \\
& \leq(b-a)\left(d N_{1}+k Z N\right)\left|u\left(x_{1}, \jmath_{1}\right)-u^{*}\left(x_{1}, \jmath_{1}\right)\right| \\
& =\Gamma\left|u\left(x_{1}, \jmath_{1}\right)-u^{*}\left(x_{1}, \jmath_{1}\right)\right|,
\end{aligned}
$$

from which we get $(1-\Gamma)\left|u-u^{*}\right| \leq 0$. Since $0<\Gamma<1$, therefore $\left|u-u^{*}\right|=0$. Therefore, $u=u^{*}$ and this completes the proof.

Theorem 2. The series solution $u\left(x_{1}, \jmath_{1}\right)=\sum_{i=0}^{\infty} u_{i}\left(x_{1}, \jmath_{1}\right)$ of (1) using HPM convergence when $0<\Gamma<1$ and $\left\|u_{1}\left(x_{1}, j_{1}\right)\right\|<$ $\infty$.

Proof. Denote with $(C[\dot{J}],\|\cdot\|)$ the Banach space of all continuous functions on $\dot{J}$ with the norm $\left\|\dot{f}\left(x_{1}, j_{1}\right)\right\|=$ $\max \left|\dot{f}\left(x_{1}, \jmath_{1}\right)\right|$ for all $x_{1}, \jmath_{1}$ in $\dot{J}$. Define the sequence of partial sums $\delta_{n}$, and let $\delta_{n}$ and $\delta_{m}$ be arbitrary partial sums with $n \geq m$. We are going to prove that $\delta_{n}=\sum_{i=0}^{n} u_{i}\left(x_{1}, \jmath_{1}\right)$ is a Cauchy sequence in this Banach space:

$$
\begin{aligned}
& \left\|\delta_{n}-\delta_{m}\right\|=\max _{\forall x_{1}, j_{1} \in j}\left|\delta_{n}-\delta_{m}\right| \\
& =\max _{\forall x_{1}, j_{1} \in \dot{J}}\left|\sum_{i=m+1}^{n} u_{i}\left(x_{1}, \jmath_{1}\right)\right| \\
& =\max _{\forall x_{1}, j_{1} \in \dot{J}} \mid \sum_{i=m+1}^{n}\left[\int_{a}^{x_{1}} k_{1}\left(x_{1}, \jmath_{1}\right) \bar{H}_{i} d \tau\right. \\
& \left.-\sum_{j=0}^{k-1} \int_{a}^{x_{1}} k_{2}\left(x_{1}, \jmath_{1}\right) L_{i_{j}} d j_{1}\right] \mid \\
& =\max _{\forall x_{1}, j_{1} \in \dot{J}} \mid \int_{a}^{x_{1}} k_{1}\left(x_{1}, \jmath_{1}\right)\left(\sum_{i=m}^{n-1} \bar{H}_{i}\right) d \tau \\
& -\sum_{j=0}^{k-1} \int_{a}^{x_{1}} k_{2}\left(x, \jmath_{1}\right)\left(\sum_{i=m}^{n-1} L_{i_{j}}\right) d \jmath_{1} \mid .
\end{aligned}
$$

From (20), we have

$$
\begin{aligned}
& \sum_{i=m}^{n-1} \bar{H}_{i}=\gamma\left(\delta_{n-1}\right)-\gamma\left(\delta_{m-1}\right) \\
& \sum_{i=m}^{n-1} L_{i_{j}}=D^{j}\left(\delta_{n-1}\right)-D^{j}\left(\delta_{m-1}\right) .
\end{aligned}
$$


So,

$$
\begin{aligned}
& \left\|\delta_{n}-\delta_{m}\right\| \\
& =\max _{\forall x_{1}, j_{1} \in \dot{J}}\left(\mid \int_{a}^{x_{1}} k_{1}\left(x_{1}, \jmath_{1}\right)\left[\gamma\left(\delta_{n-1}\right)-\gamma\left(\delta_{m-1}\right)\right] d \tau\right. \\
& \left.-\sum_{j=0}^{k-1} \int_{a}^{x_{1}} k_{2}\left(x_{1}, \jmath_{1}\right)\left[D^{j}\left(\delta_{n-1}\right)-D^{j}\left(\delta_{m-1}\right)\right] d \jmath_{1} \mid\right) \\
& \leq \max _{\forall x_{1}, j_{1} \in \dot{J}}\left(\int_{a}^{x_{1}}\left|k_{1}\left(x_{1}, \jmath_{1}\right)\right|\left|\gamma\left(\delta_{n-1}\right)-\gamma\left(\delta_{m-1}\right)\right| d \tau\right. \\
& \left.+\sum_{j=0}^{k-1}\left(\int_{a}^{x_{1}}\left|k_{2}\left(x_{1}, \jmath_{1}\right)\right|\left|D^{j}\left(\delta_{n-1}\right)-D^{j}\left(\delta_{m-1}\right)\right| d \jmath_{1}\right)\right) \\
& \leq \Gamma\left\|\delta_{n-1}-\delta_{m-1}\right\| .
\end{aligned}
$$

Let $n=m+1$; then,

$$
\begin{aligned}
\left\|\delta_{m+1}-\delta_{m}\right\| & \leq \Gamma\left\|\delta_{m}-\delta_{m-1}\right\| \leq \Gamma^{2}\left\|\delta_{m-1}-\delta_{m-2}\right\| \\
& \leq \cdots \leq \Gamma^{m}\left\|\delta_{1}-\delta_{0}\right\| .
\end{aligned}
$$

So,

$$
\begin{aligned}
\left\|\delta_{n}-\delta_{m}\right\| \leq & \left\|\delta_{m+1}-\delta_{m}\right\|+\left\|\delta_{m+2}-\delta_{m+1}\right\|+\cdots \\
& +\left\|\delta_{n}-\delta_{n-1}\right\| \\
\leq & {\left[\Gamma^{m}+\Gamma^{m+1}+\cdots+\Gamma^{n-1}\right]\left\|\delta_{1}-\delta_{0}\right\| } \\
\leq & \Gamma^{m}\left[1+\Gamma+\Gamma^{2}+\cdots+\Gamma^{n-m-1}\right]\left\|\delta_{1}-\delta_{0}\right\| \\
\leq & \Gamma^{m}\left[\frac{1-\Gamma^{n-m}}{1-\Gamma}\right]\left\|u_{1}\left(x_{1}, j_{1}\right)\right\| .
\end{aligned}
$$

Since $0<\Gamma<1$, we have $\left(1-\Gamma^{n-m}\right)<1$; then,

$$
\left\|\delta_{n}-\delta_{m}\right\| \leq \frac{\Gamma^{m}}{1-\Gamma} \max _{\forall x_{1}, j_{1} \in j}\left\|u_{1}\left(x_{1}, \jmath_{1}\right)\right\| .
$$

But $\left|u_{1}\left(x_{1}, j_{1}\right)\right|<\infty$ (since $\dot{f}_{1}\left(x_{1}, \jmath_{1}\right)$ is bounded), so, as $m \rightarrow \infty$, then $\left\|\delta_{n}-\delta_{m}\right\| \rightarrow 0$. We conclude that $\delta_{n}$ is a Cauchy sequence in $C[J]$, and therefore the series is convergent and the proof is complete.

Theorem 3. When using VIM for solving two-dimensional nonlinear VFIDE where $0<\Gamma<1$ and $P_{k}\left(x_{1}, j_{1}\right)=1$, then $u\left(x_{1}, j_{1}\right)=\lim _{n \rightarrow \infty} u_{n}\left(x_{1}, j_{1}\right)$ converges.

Proof. One has

$$
\begin{gathered}
u_{n+1}\left(x_{1}, \jmath_{1}\right)=u_{n}\left(x_{1}, \jmath_{1}\right)-\int_{0}^{j_{1}}\left[u_{n}\left(x_{1}, \varsigma\right)\right. \\
-f_{1}\left(x_{1}, \varsigma\right)-\int_{a}^{x_{1}} k_{1}\left(x_{1}, \varsigma\right) \gamma\left(u_{n}^{l}\left(x_{1}, \varsigma\right)\right) d \tau \\
\left.+\sum_{j=0}^{k-1} \int_{a}^{x_{1}} k_{2}\left(x_{1}, \varsigma\right)\left(u_{n}\right)^{j}\left(x_{1}, \varsigma\right) d \jmath_{1}\right] d \varsigma
\end{gathered}
$$

$$
\begin{aligned}
& u\left(x_{1}, \jmath_{1}\right)=u\left(x_{1}, \jmath_{1}\right)-\int_{0}^{j_{1}}\left[u\left(x_{1}, \varsigma\right)-f_{1}\left(x_{1}, \varsigma\right)\right. \\
& -\int_{a}^{x_{1}} k_{1}\left(x_{1}, \varsigma\right) \gamma\left(u^{l}\left(x_{1}, \varsigma\right)\right) d \tau \\
& \left.+\sum_{j=0}^{k-1} \int_{a}^{x_{1}} k_{2}\left(x_{1}, \varsigma\right)(u)^{j}\left(x_{1}, \varsigma\right) d \jmath_{1}\right] d \varsigma .
\end{aligned}
$$

By subtracting relation (41) from (42),

$$
\begin{aligned}
& u_{n+1}\left(x_{1}, \jmath_{1}\right)-u\left(x_{1}, \jmath_{1}\right)=u_{n}\left(x_{1}, \jmath_{1}\right)-u\left(x_{1}, \jmath_{1}\right)-\int_{0}^{\jmath_{1}}\left[u_{n}\left(x_{1}, \varsigma\right)-u\left(x_{1}, \varsigma\right)\right. \\
& \left.-\int_{a}^{x_{1}} k_{1}\left(x_{1}, \varsigma\right)\left[\gamma\left(u_{n}^{l}\left(x_{1}, \varsigma\right)\right)-\gamma\left(u^{l}\left(x_{1}, \varsigma\right)\right)\right] d \tau+\sum_{j=0}^{k-1} \int_{a}^{x_{1}} k_{2}\left(x_{1}, \varsigma\right)\left[D^{j}\left(u_{n}\left(x_{1}, \varsigma\right)\right)-D^{j}\left(u\left(x_{1}, \varsigma\right)\right) d \jmath_{1}\right]\right] d \varsigma .
\end{aligned}
$$

Hence, we set

$$
\begin{gathered}
e_{n+1}\left(x_{1}, \jmath_{1}\right)=u_{n+1}\left(x_{1}, \jmath_{1}\right)-u\left(x_{1}, \jmath_{1}\right), \\
e_{n}\left(x_{1}, \jmath_{1}\right)=u_{n}\left(x_{1}, \jmath_{1}\right)-u\left(x_{1}, \jmath_{1}\right) .
\end{gathered}
$$

Then,

$$
\begin{array}{r}
e_{n+1}\left(x_{1}, \jmath_{1}\right)=e_{n}\left(x_{1}, \jmath_{1}\right)-\int_{0}^{\jmath_{1}}\left[u_{n}\left(x_{1}, \varsigma\right)-u\left(x_{1}, \varsigma\right)\right. \\
-\int_{a}^{x_{1}} k_{1}\left(x_{1}, \varsigma\right)\left[\gamma\left(u_{n}^{l}\left(x_{1}, \varsigma\right)\right)-\gamma\left(u^{l}\left(x_{1}, \varsigma\right)\right)\right] d \tau
\end{array}
$$

$$
+\sum_{j=0}^{k-1} \int_{a}^{x_{1}} k_{2}\left(x_{1}, \varsigma\right)
$$$$
\left.\cdot\left[D^{j}\left(u_{n}\left(x_{1}, \varsigma\right)\right)-D^{j}\left(u\left(x_{1}, \varsigma\right)\right)\right] d \jmath_{1}\right] d \varsigma
$$$$
\leq e_{n}\left(x_{1}, \jmath_{1}\right)\left(1-(b-a)\left(d N_{1}+k Z N\right)\right)=(1-\Gamma)
$$$$
\cdot e_{n}\left(x_{1}, \jmath_{1}\right) \text {. }
$$ 
Therefore,

$$
\left\|e_{n+1}\right\|=\max _{\forall x_{1}, j_{1} \in J}\left|e_{n+1}\right| \leq(1-\Gamma) \max _{\forall x_{1}, J_{1} \in \dot{J}}\left|e_{n}\right|=\left\|e_{n}\right\| .
$$

Since $0<\Gamma<1$, then $\left\|e_{n}\right\| \rightarrow 0$. So, the series converges and the proof is complete.

Theorem 4. The maximum absolute truncation error of the series $u\left(x_{1}, j_{1}\right)=\sum_{i=0}^{\infty} u_{i}\left(x_{1}, j_{1}\right)$ to (1) is estimated to be

$$
\begin{gathered}
\max _{\forall x_{1}, j_{1} \in \hat{J}}\left|u\left(x_{1}, \jmath_{1}\right)-\sum_{i=0}^{m} u_{i}\left(x_{1}, \jmath_{1}\right)\right| \\
\leq \frac{\Gamma^{m+1}}{1-\Gamma} \max _{\forall x_{1}, j_{1} \in \dot{J}}\left|\gamma\left(u_{0}\right)\right| .
\end{gathered}
$$

Proof. From Theorem 2 and inequality (40), we have

$$
\left\|\delta_{n}-\delta_{m}\right\| \leq \frac{\Gamma^{m}}{1-\Gamma} \max _{\forall x_{1}, j_{1} \in J}\left\|u_{1}\left(x_{1}, \jmath_{1}\right)\right\| .
$$

As $n \rightarrow \infty$, then $\delta_{n} \rightarrow u\left(x_{1}, j_{1}\right)$ and we have

$$
\begin{aligned}
& \max _{\forall x, t \in J}\left|u_{1}\left(x_{1}, \jmath_{1}\right)\right| \\
& \quad \leq(b-a)\left(d N_{1}+k Z N\right) \max _{\forall x_{1}, j_{1} \in J}\left|\gamma\left(u_{0}\right)\right| \\
& \quad \leq \Gamma \max _{\forall x_{1},,_{1} \in \dot{J}}\left|\gamma\left(u_{0}\right)\right| .
\end{aligned}
$$

So,

$$
\left\|u\left(x_{1}, j_{1}\right)-\delta_{m}\right\| \leq \frac{\Gamma^{m+1}}{1-\Gamma} \max _{\forall x_{1}, j_{1} \in \dot{J}}\left|\gamma\left(u_{0}\right)\right| .
$$

Finally, the maximum absolute truncation error in the interval $J$ is

$$
\begin{aligned}
& \max _{\forall x_{1}, j_{1} \in \dot{J}}\left|u\left(x_{1}, \jmath_{1}\right)-\sum_{i=0}^{m} u_{i}\left(x_{1}, \jmath_{1}\right)\right| \\
& \leq \frac{\Gamma^{m+1}}{1-\Gamma} \max _{\forall x_{1}, j_{1} \in \dot{J}}\left|\gamma\left(u_{0}\right)\right| .
\end{aligned}
$$

\section{Numerical Examples}

Example 1. Consider the nonlinear integrodifferential equation

$$
\begin{aligned}
& \frac{\partial^{2} u\left(x_{1}, \jmath_{1}\right)}{\partial j_{1}^{2}}+\frac{\partial^{2} u\left(x_{1}, \jmath_{1}\right)}{\partial x_{1} \partial j_{1}}-x_{1} u^{3}\left(x_{1}, \jmath_{1}\right) \\
& \quad+\int_{0}^{x_{1}} \int_{0}^{1} u^{2}(y, \tau) d y d \tau=\dot{f}\left(x_{1}, \jmath_{1}\right),
\end{aligned}
$$

$$
x_{1} \in[0,1],
$$

where

$$
\dot{f}\left(x_{1}, \jmath_{1}\right)=2 \jmath_{1}+\frac{1}{15} x_{1}^{3} \jmath_{1}^{5}-x_{1}^{4} \jmath_{1}^{5}+2 x_{1},
$$

with the initial conditions

$$
\text { I.Cs : }\left\{\begin{array}{l}
u\left(x_{1}, 0\right)=0, \\
\frac{\partial u\left(x_{1}, 0\right)}{\partial \jmath_{1}}=0,
\end{array}\right.
$$

which has exact solution $u\left(x_{1}, t_{1}\right)=x_{1} j_{1}^{2}$. This example is solved by using the variational iteration method with He's polynomials VHPM (see (26)) and VAHPM (see (27)) expressing the nonlinear terms of $\breve{H}$ and $\bar{H}$, respectively, in Table 1.

Example 2. Consider the nonlinear integrodifferential equation

$$
\begin{array}{r}
u\left(x_{1}, \jmath_{1}\right) \frac{\partial^{2} u\left(x_{1}, \jmath_{1}\right)}{\partial \jmath_{1}^{2}}-4 u\left(x_{1}, \jmath_{1}\right) \frac{\partial^{2} u\left(x_{1}, \jmath_{1}\right)}{\partial x_{1}^{2}} \\
+4 \int_{0}^{x_{1}} \int_{0}^{1} u^{2}(y, \tau) d y d \tau=\dot{f}\left(x_{1}, \jmath_{1}\right), \\
x_{1} \in[0,1],
\end{array}
$$

where

$$
\begin{aligned}
\dot{f} & \left(x_{1}, \jmath_{1}\right) \\
& =\left(x_{1}-\frac{1}{2 \pi} \sin \left(2 \pi x_{1}\right)\right)\left(\jmath_{1}-\frac{1}{4 \pi} \sin \left(4 \pi \jmath_{1}\right)\right),
\end{aligned}
$$

with the boundary conditions

$$
\text { B.Cs : } u\left(0, \jmath_{1}\right)=u\left(1, \jmath_{1}\right)=0
$$

and the initial conditions

$$
\text { I.Cs : } \begin{cases}u\left(x_{1}, 0\right)=\sin \left(\pi x_{1}\right), & 0 \leq x_{1} \leq 1 \\ \frac{\partial u\left(x_{1}, 0\right)}{\partial \jmath_{1}}=0, & 0 \leq x_{1} \leq 1,\end{cases}
$$

which has exact solution $u\left(x_{1}, \jmath_{1}\right)=\sin \left(\pi x_{1}\right) \cos \left(2 \pi \jmath_{1}\right)$. This example is solved by using VHPM (see (26)) and VAHPM (see (27)) expressing the nonlinear terms of $\breve{H}$ and $\bar{H}$, respectively, in Table 2 .

\section{Conclusion}

In this paper, we applied VHPM and VAHPM to solve nonlinear mixed VFIDE. The proposed VAHPM converges faster than the VHPM. Based on the proposed formula (27) with accelerated He's polynomials formula (20), the convergence of the technique is proved. The presented technique is very easy to implement and it reduces the computation size. 
TABLE 1: Exact solution, approximate solution, and error by using VHPM and VAHPM.

\begin{tabular}{lcccccc}
\hline$x_{1}$ & $J_{1}$ & Exact & Appr. $_{(\mathrm{VHPM})}$ & Err. $_{\text {(VHPM })}$ & Appr. $_{\text {(VAHPM })}$ & Err. ${ }_{(\mathrm{VAHPM})}$ \\
\hline $0.00 E+00$ & $0.00 E+00$ & $0.00000 E+00$ & $0.00000 E+00$ & $0.00000 E+00$ & $0.00000 E+00$ & $0.00000 E+00$ \\
$3.00 E-03$ & $3.00 E-03$ & $2.70000 E-08$ & $9.90000 E-08$ & $2.70000 E-08$ & $3.60000 E-08$ & $9.00000 E-09$ \\
$5.00 E-03$ & $5.00 E-03$ & $1.25000 E-07$ & $4.58330 E-07$ & $3.33333 E-07$ & $1.66667 E-07$ & $4.16670 E-08$ \\
$7.00 E-03$ & $7.00 E-03$ & $3.43000 E-07$ & $1.25767 E-06$ & $9.14670 E-07$ & $4.57333 E-07$ & $1.14333 E-07$ \\
$9.00 E-03$ & $9.00 E-02$ & $7.290000 E-07$ & $2.67300 E-06$ & $1.94400 E-06$ & $9.72000 E-07$ & $2.43000 E-07$ \\
\hline
\end{tabular}

TABLE 2: Exact solution, approximate solution, and error by using VHPM and VAHPM.

\begin{tabular}{lcccccc}
\hline$x_{1}$ & $J_{1}$ & Exact & Appr. ${ }_{(\mathrm{VHPM})}$ & Err. $_{\text {(VHPM })}$ & Appr. $_{\text {(VAHPM })}$ & Err. ${ }_{(\mathrm{VAHPM})}$ \\
\hline $0.00 E+00$ & $0.00 E+00$ & $0.00000 E+00$ & $0.00000 E+00$ & $0.00000 E+00$ & $0.00000 E+00$ & $0.00000 E+00$ \\
$3.00 E-03$ & $3.00 E-03$ & $9.42295 E-03$ & $9.41128 E-03$ & $1.16700 E-05$ & $9.42443 E-03$ & $1.48000 E-06$ \\
$5.00 E-03$ & $5.00 E-03$ & $1.56997 E-02$ & $1.56384 E-02$ & $6.13000 E-05$ & $1.57054 E-02$ & $5.70000 E-06$ \\
$7.00 E-03$ & $7.00 E-03$ & $2.19680 E-02$ & $2.17975 E-02$ & $1.70500 E-04$ & $2.19817 E-02$ & $1.37000 E-05$ \\
$9.00 E-03$ & $9.00 E-02$ & $2.82253 E-02$ & $2.78610 E-02$ & $3.64300 E-04$ & $2.82534 E-02$ & $2.81000 E-05$ \\
\hline
\end{tabular}

\section{Conflicts of Interest}

The authors declare that they have no conflicts of interest.

\section{References}

[1] J. Huesin, A. Omar, and S. AL-shara, "Numerical solution of linear integro-differential equations," Journal of Mathematics and Statistics, vol. 4, no. 4, pp. 250-254, 2008.

[2] E. Babolian, Z. Masouri, and S. Hatamzadeh-Varmazyar, "NEW direct method to solve nonlinear volterra-fredholm integral and integro-differential equations using operational matrix with block-pulse functions," Progress In Electromagnetics Research B, vol. 8, pp. 59-76, 2008.

[3] A. Al-Jubory, Some Approximation Methods for Solving VolterraFredholm Integral and Integro- Differential Equations [Ph.D. thesis], University of Technology, 2010.

[4] M. Dadkhah, M. T. Kajani, and S. Mahdavi, Numerical Solution of Nonlinear Fredholm-Volterra Integro- Differential Equations Using Legendre Wavelets, ICMA, 2010.

[5] M. Rabbani and S. H. Kiasoltani, "Solving of nonlinear system of volterra-fredholm integro-differential equations by using discrete collocation method," The Journal of Mathematics and Computer Science, vol. 3, no. 4, pp. 382-387, 2011.

[6] H. D. Gherjalar and H. Mohammadikia, "Numerical solution of functional integral and integro-differential equations by using b-splines," Applied Mathematics, vol. 03, no. 12, pp. 1940-1944, 2012.

[7] J.-H. He, "The variational iteration method for eighth-order initial-boundary value problems," Physica Scripta. An International Journal for Experimental and Theoretical Physics, vol. 76, no. 6, pp. 680-682, 2007.

[8] J. He, "Variational iteration method-a kind of non-linear analytical technique: some examples," International Journal of Non-Linear Mechanics, vol. 34, no. 4, pp. 699-708, 1999.

[9] J.-H. He, "Homotopy perturbation technique," Computer Methods in Applied Mechanics and Engineering, vol. 178, no. 3-4, pp. 257-262, 1999.

[10] A. Ghorbani, "Beyond Adomian polynomials: He polynomials," Chaos, Solitons and Fractals, vol. 39, no. 3, pp. 1486-1492, 2009.
[11] I. L. Kalla, "An accelerated homotopy perturbation method for solving nonlinear equations," Journal of Fractional Calculus and Applications, vol. 3(S), pp. 1-8, 2012. 


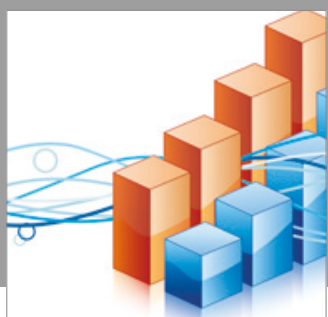

Advances in

Operations Research

vatersals

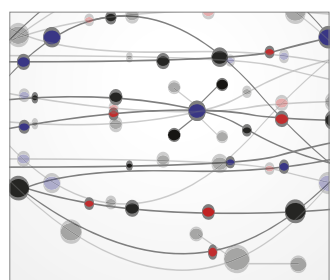

\section{The Scientific} World Journal
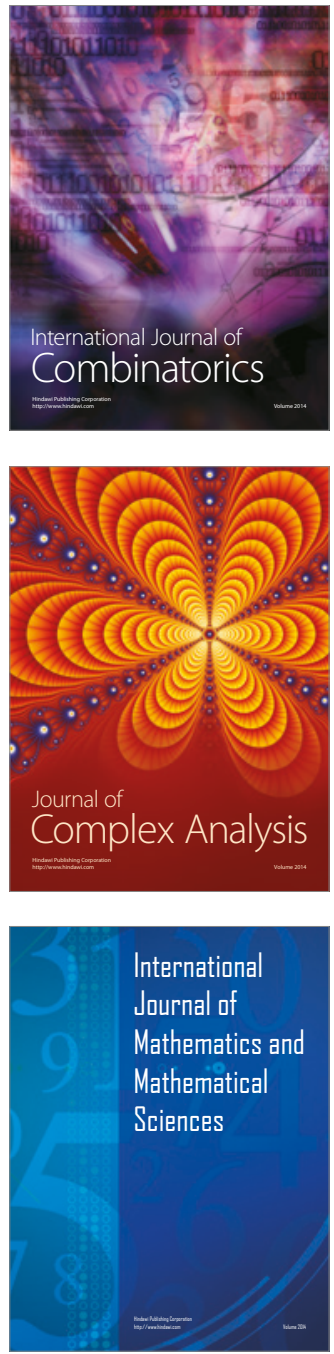
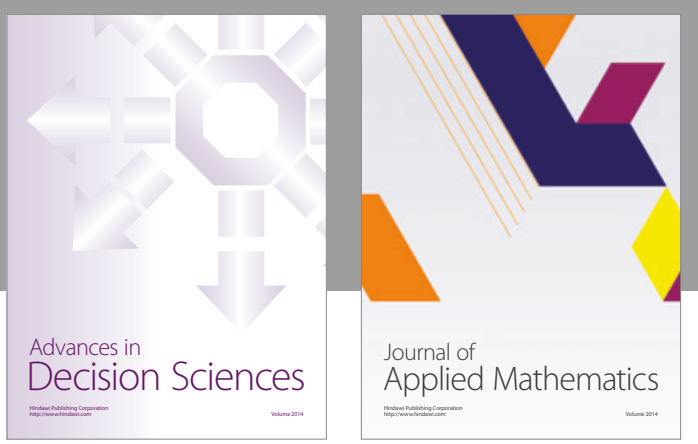

Algebra

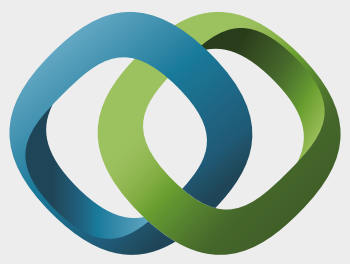

\section{Hindawi}

Submit your manuscripts at

https://www.hindawi.com
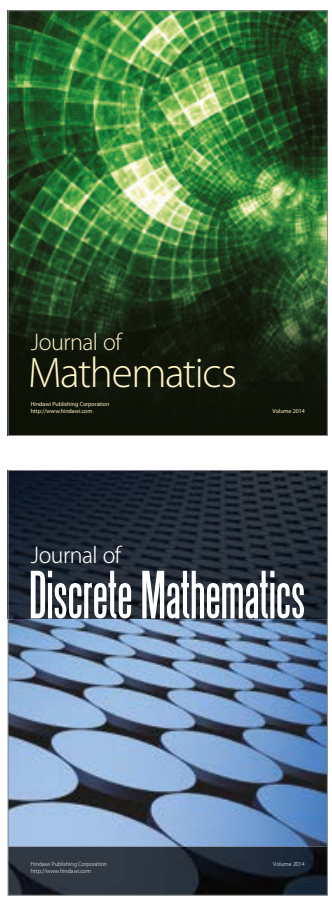

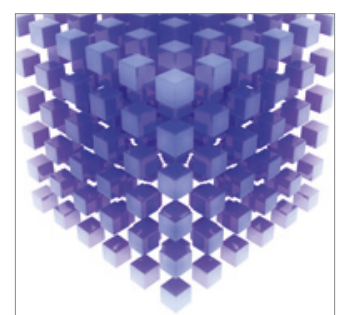

Mathematical Problems in Engineering
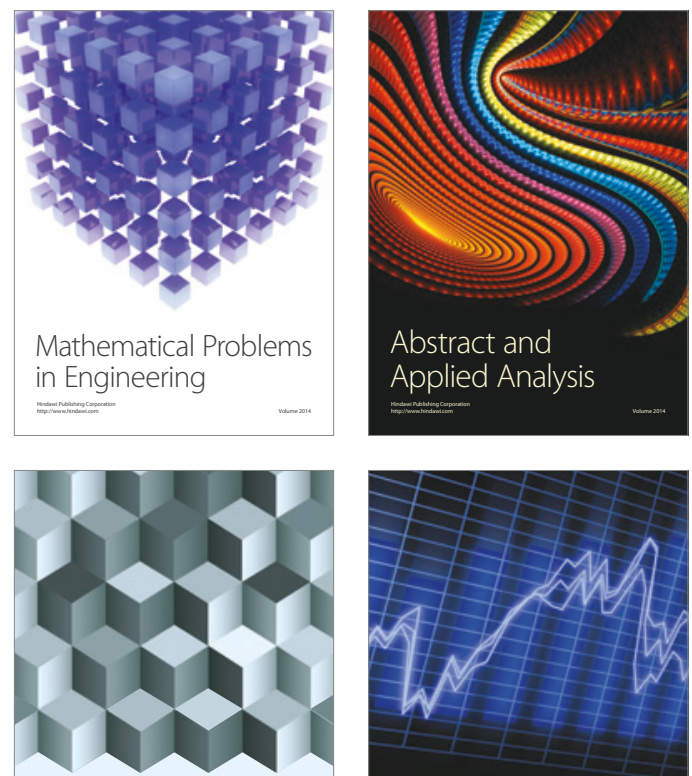

Journal of

Function Spaces

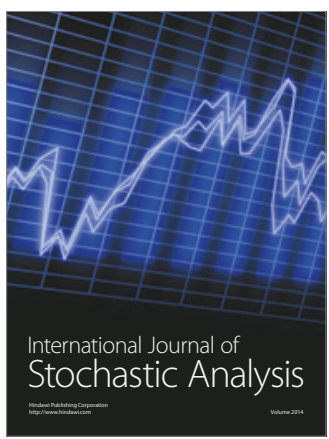

Probability and Statistics
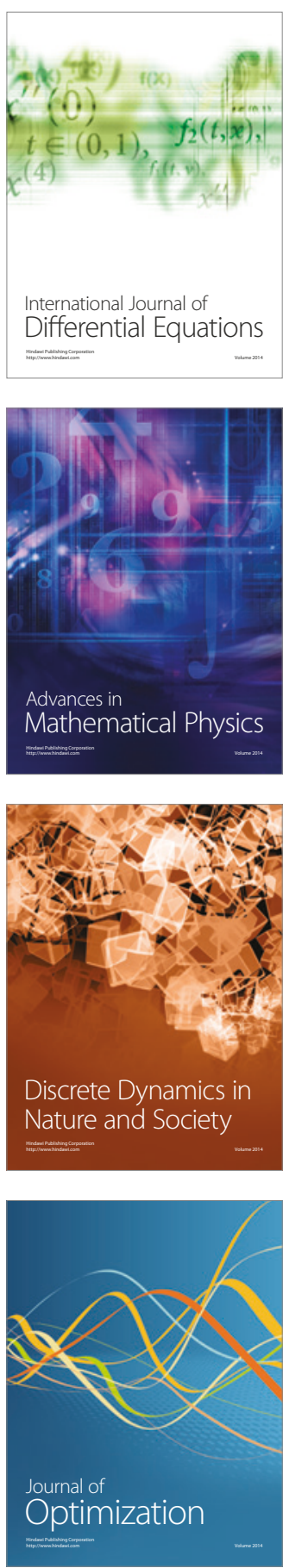